\title{
Article \\ Thermodynamics and Kinetics Research of the Fluorination Process of the Concentrate Rutile
}

\author{
Vladimir A. Karelin ${ }^{1}$, Son Hai Le ${ }^{1,2}{ }^{10}$, Nadezhda V. Karelina ${ }^{1}$, Alexander N. Strashko ${ }^{1}$, Alexander V. Sazonov ${ }^{1}$ \\ and Huong M. T. Le ${ }^{3, *}$ \\ 1 Division for Nuclear-Fuel Cycle, School of Nuclear Science and Engineering, National Research Tomsk \\ Polytechnic University, Lenin Ave., 30, 634050 Tomsk, Russia; vakarelin@tpu.ru (V.A.K.); \\ lehaison@tpu.ru (S.H.L.); nvk15@tpu.ru (N.V.K.); strashkoan@tpu.ru (A.N.S.); srv@tpu.ru (A.V.S.) \\ 2 Center for Rare Elements' Technology, Institute for Technology of Radioactive and Rare Earth \\ Elements (ITRRE), Vietnam Atomic Energy Institute (VINATOM), 48 Lang Ha, Dong Da, \\ Hanoi 11513, Vietnam \\ 3 Division of Training and Technology Transfer, Nuclear Training Center (NTC), Vietnam Atomic Energy \\ Institute (VINATOM), 140 Nguyen Tuan, Thanh Xuan, Hanoi 11416, Vietnam \\ * Correspondence: lethimaihuong@vinatom.gov.vn
}

Citation: Karelin, V.A.; Le, S.H.; Karelina, N.V.; Strashko, A.N.; Sazonov, A.V.; Le, H.M.T.

Thermodynamics and Kinetics Research of the Fluorination Process of the Concentrate Rutile. Metals 2022, 12, 34. https://doi.org/10.3390/ met12010034

Academic Editor: Silvie Brožová

Received: 12 November 2021

Accepted: 20 December 2021

Published: 24 December 2021

Publisher's Note: MDPI stays neutral with regard to jurisdictional claims in published maps and institutional affiliations.

Copyright: (C) 2021 by the authors. Licensee MDPI, Basel, Switzerland. This article is an open access article distributed under the terms and conditions of the Creative Commons Attribution (CC BY) license (https:// creativecommons.org/licenses/by/ $4.0 /)$.

\begin{abstract}
The growth in the production of titanium metal and its compounds leads to an increase in the amount of toxic waste. As a result, at the legislative level, emissions of such wastes are limited, which leads to a drop in the production of titanium-containing products and a shortage of titanium in the international market. This paper presents the results of the process of fluorination of rutile concentrate from the Tarsky deposit (Russia, Omsk region) with elemental fluorine using a laboratory setup of a special design. For fluorination, samples of rutile concentrate weighing $0.1-1.0 \mathrm{~g}$ were used. The particle size distribution of particles varied from $2 \times 10^{-6}$ to $2 \times 10^{-5} \mathrm{~m}$. To determine the possibility of carrying out the process, the calculation of the change in the logarithm of the equilibrium constant versus temperature was performed. The influence of the following operating parameters on the fluorination process has been studied: various concentrations of $\mathrm{F}_{2}$ in a fluorinating mixture of fluorine with nitrogen; process time from 0 to $9 \mathrm{~min}$; different ratios of the initial solid phase to fluorine (10 and 50\% excess of fluorine and 10 and $50 \%$ of its deficiency); fluorination temperature in the range of $300-1800 \mathrm{~K}$. A kinetic equation is selected that most accurately describes the fluorination process. The values of the activation energy and the preexponential factor in the kinetic equation are determined. The obtained results show that with an increase in the fluorine content in the fluorinating gas mixture and the temperature of the process, the fluorination rate increases. Optimal conditions for fluorination: temperature $-680 \mathrm{~K}$; time -5 min excess fluorine in the fluorinating mixture-20-25\%. The obtained results allow to propose and consider the conditions of process execution on industrial equipment.
\end{abstract}

Keywords: rutile concentrate; fluorination process; titanium tetra fluoride; admixtures fluorides; thermodynamics and kinetics of process

\section{Introduction}

With the development of science-intensive technologies, the need for the creation of structural materials that work in critical conditions is sharply increasing. These materials include titanium alloys. The creation of technological processes allowing to ensure high quality of titanium alloys and their minimum cost is the key task of technologies for obtaining high-tech structural materials. The development of modern technologies for the chemical processing of titanium concentrates and the synthesis of high-purity metallic titanium is one of the main processes preceding the creation of titanium alloys, since the physicochemical properties and cost of products made of titanium and its alloys depend on the content of impurities in a titanium product. 
Currently, two main methods are used in industry for processing titanium-containing concentrates: sulfate (sulfuric acid) and chloride. The main problem, as well as the disadvantage of sulfate technology, is environmental pollution due to the need to discharge a large amount of waste in the form of $\mathrm{CaSO}_{4}$ into the environment. As a result, the production cost of titanium dioxide using sulfate technology is very high. However, the sulfate method has the advantage that it does not require a high titanium feedstock [1]. The chloride method is considered to be more advanced and environmentally friendly. However, the main disadvantage of the method is that a large amount of gaseous, liquid and solid toxic chloride waste is discharged, and the total amount of titanium extracted into pure titanium chloride does not exceed $89 \%$ of the mass. In addition, for the chlorination process to be highly efficient, the titanium content $\left(\mathrm{as}_{\mathrm{TiO}}\right)$ in the starting material must be high $(>90 \%)$. In this case, it becomes necessary to carry out auxiliary operations for the purification of raw materials and the recycling process in order to increase the titanium content in the raw materials to a level corresponding to technological requirements. As a result, only 10 to $50 \%$ of titanium metal can be obtained from raw materials, which significantly increases its cost [2].

The current production rate of titanium dioxide in the world is about 2750 thousand tons/year, corresponding to the amount of harmful solid waste by chlorination method of about 1467 thousand tons/year (under the condition that all factories are equal, switch to chloride technology) [3,4].

In the last decade, the development of the sulfate method associated with the introduction of various reagents (additives) sulfuric acid. For example, in [5] a water technology is described, in which it is recommended to use a mixture of $\mathrm{H}_{2} \mathrm{SO}_{4}(10 \%$ vol.)-HF (15\% vol.) to open titanium ores. At opening, $\mathrm{TiF}_{6}{ }^{2-}$ is first formed, which, upon subsequent interaction with $\mathrm{H}_{2} \mathrm{SO}_{4}$, forms $\mathrm{Ti}\left(\mathrm{SO}_{4}\right)_{2}$ [5]. In [6-8], the process of sulfate-fluoride leaching of titanium from ilmenite and the formation of fluorotitanate ions in aqueous solutions and the subsequent synthesis of $\mathrm{Ti}\left(\mathrm{SO}_{4}\right)_{2}$ in a sulfuric acid medium is proposed. In fact, this is a modification of the well-known sulfate technology of titanium ore opening. The main disadvantage of this technology is the formation of a large amount of calcium sulfate, which must be separated from production waste and spend significant funds on its storage.

An aquatic technology is being developed for opening titanomagnetite ore by selective extraction of titanium with solutions containing ammonium fluoride (ammonium and fluorine ions) [9]. A technology based on the use of metal and ammonium hydrofluorides for opening titanium ores and concentrates is presented [10]. As a result of the interaction of titanium and iron oxides with aqueous solutions of ammonium fluoride, aqueous solutions of HF (hydrofluoric acid) are formed, which, under the conditions of the process, have a strong corrosive effect on the material of the apparatus. This does not allow the use of this method in an industrial environment.

A high-temperature technology for the synthesis of titanium slag based on $\mathrm{TiO}_{2}$, suitable for metallothermal reduction and synthesis of metallic titanium, has been described [11]. However, if magnesium is used as a metal-reducing agent in the reduction in slag, then this is actually the well-known Kroll process, which is the only method used in industrial conditions to obtain titanium sponge.

The process of synthesis of rutile from titanium slags from the Panzhihua deposit (China) by oxidative roasting of these slags at $1100^{\circ} \mathrm{C}$ for $2 \mathrm{~h}$ is presented [12]. However, this method is not new since it is described in the preparation of various titanium ores for the Kroll process-the reduction in $\mathrm{TiO}_{2}$ with magnesium.

Recently, various types of titanium electrolytic reduction processes in fluoride-chloridesalt melts have been intensively developed. $\mathrm{TiO}_{2}$ is used as a starting material. These are FFC, MER-, PRP-, Osaka-, Suzuki- and Chinuka-processes [13-18]. However, these processes have not yet passed the stage of laboratory tests and research using "pilot" installations.

In the near future, we can expect a breakthrough in the creation of new titanium technologies, which will significantly reduce its prime cost. In this case, the cost of titanium 
can become commensurate with the cost of stainless steel. As a result, titanium consumption could skyrocket and replace stainless steel and other materials in the global market.

In the opinion of the authors of the article, in order to expand the areas of application of titanium and its alloys, it is necessary to develop a technology that makes it possible to effectively separate titanium from impurities, and then convert the volatile titanium compound into metallic titanium (powder or sponge). The number of stages of such a process should be minimal (no more than 3-4 stages). Such a process can be the process of direct fluorination of titanium concentrates with elemental fluorine, which makes it possible to transfer titanium into a gaseous compound, for example, $\mathrm{TiF}_{4}$, and at the same time effectively separate non-volatile impurity fluorides from $\mathrm{TiF}_{4}$. Subsequently, the reduction in $\mathrm{TiF}_{4}$ should be carried out in a high-frequency pulse discharge, and the resulting elemental fluorine should be recycled again into the fluorination process. In order to study the mechanism of chemical processes occurring in this proposed technology, it is necessary to start from basic studies, namely thermodynamic and kinetic.

In this paper, a method of processing titanium-containing minerals is introduced by fluorine technology with rutile as the raw material, and some thermodynamic and kinetic parameters are calculated to evaluate and propose a schematic diagram of a facility for fluorination of rutile concentrate in industrial conditions.

\section{Materials and Methods}

\subsection{Materials}

All Rutile concentrates used in the experiment were selected and refined with high $\mathrm{TiO}_{2}$ content from the Tasky region (Omsk), Russiawhich took place was described in our previous Procedia Chemistry conference report [19]. The refining process is a heat treatment process so there are no loss on ignition (loss of mass on heating) and volatiles.These concentrates have the following composition, \% (mass): $93.2 \mathrm{TiO}_{2} ; 1.8 \mathrm{SiO}_{2} ; 2.7 \mathrm{FeO}+\mathrm{Fe}_{2} \mathrm{O}_{3}$; $1.0 \mathrm{Al}_{2} \mathrm{O}_{3} ; 0.6 \mathrm{MnO} ; 0.4 \mathrm{CaO} ; 0.2 \mathrm{MgO} ; 0.1 \mathrm{~V}_{2} \mathrm{O}_{5}$. The total content of the main components reaches $100 \%$, which means that it does not contain other impurities. Pure fluorine $\left(\mathrm{F}_{2}\right)$ gas is used for fluorination, gaseous Ar are supplied to create an inert environment for the reaction to take place unaffected.

\subsection{Experiment}

The fluorination technology was discovered with the main purpose to produce the final product, which is metallic titanium, with high purity. It consists of two main stages:

- The fluorination stage to obtain titanium Fluoride $\left(\mathrm{TiF}_{4}\right)$. It includes a reaction phase that forms a mixture of Fluoride products in which there are two product groups: solid products and gaseous products. Gas products: $\mathrm{TiF}_{4}, \mathrm{VF}_{5}$ (or $\mathrm{VOF}_{3}$ ) and $\mathrm{SiF}_{4}$. Remaining in the solid phase are the fluoride salts of Iron, Magnesium, Manganese, Calcium and Aluminum and the unreacted components. This mixture was cooled in stages to separate the $\mathrm{TiF}_{4}$ salts from the Fluoride salts.

- $\quad$ Electrolysis obtained metal titanium (ingot form-ingot) in Fluoride salt. This process uses a eutecticmixture of fluoride saltsof alkali metals NaF-LiF-KF.

Kinetic studies were carried out on an experimental setup, the diagram of which is shown in Figure 1. Fluorination was carried out in a vertical reactor 1 heated by a heating system 18.The process has been carried out, manufactured and equipmentinstalled. The stages and process of supplying materials and gases have been described in the previous report [19]. It includes: 


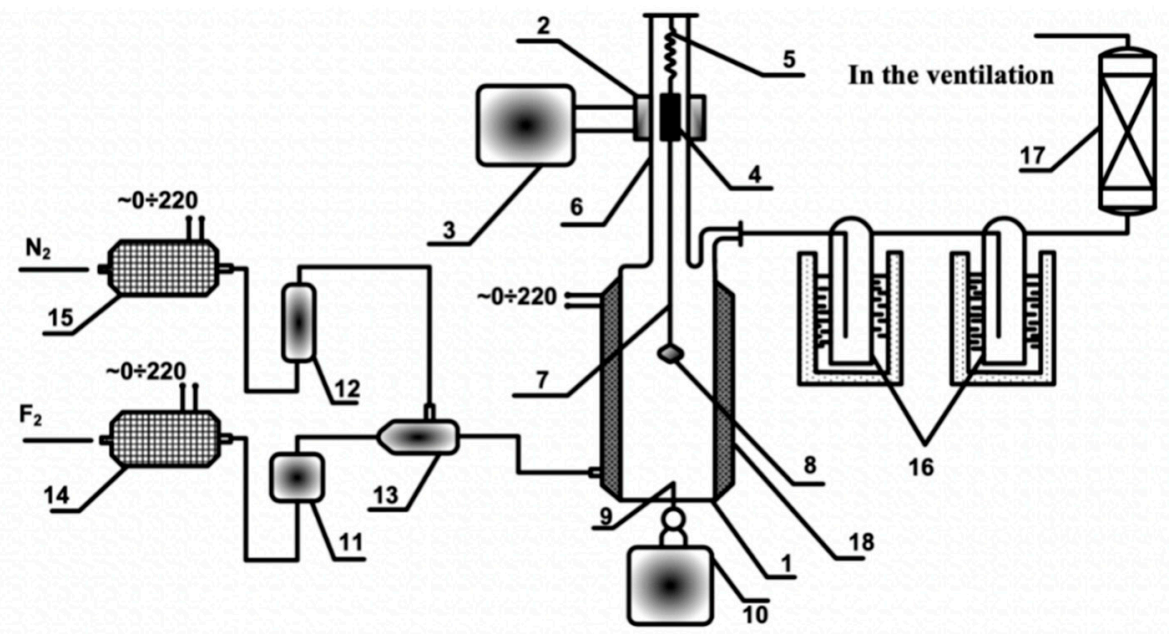

Figure 1. Diagram of setting up testing system of fluorination processing of Rutile concentrate [19]. 1-Fluorination reactor; 2-induction coil; 3-registering device; 4-electromagnetic coil; 5—spring made of Mo; 6-measuring device; 7-molybdenum string; 8-cup from a nickel foil; 9,10—system of temperature controlling and checking; 11,12 - gas charging controlling system $\mathrm{F}_{2}$ and $\mathrm{N}_{2} ; 13$-mixture of fluorine and argon supply; 14,15-heaters of fluoride and argon; 16-condensers; 17—sorption column; 18-electric heater.

Gaseous $\mathrm{N}_{2}$ (Tompromgaz, Tomsk, Russia) 15 was pumped in and mixed with gaseous $\mathrm{F}_{2}$ (Tompromgaz, Russia) 14 in gas mixer 13 (Siberian Mechanical Plant, Glazov, Russia). The amounts of mixed gases $\mathrm{N}_{2}$ and $\mathrm{F}_{2}$ were determined by measuring instruments 11 and 12 (ManoTom, Russia). This is necessary to ensure a predetermined ratio of these gases supplied to reactor 1 (Siberian Mechanical Plant, Russia). Ratio $\mathrm{N}_{2}$ and $\mathrm{F}_{2}$ depend on the research conditions. $\mathrm{F}_{2}$ is diluted with $\mathrm{N}_{2}$ so that the fluorination process does not proceed at a very high rate (in combustion mode) and kinetic data can be obtained. A sample of the investigated rutile concentrate (weighing $0.1-1 \mathrm{~g}$ ) was preliminarily placed in a cup 8 (Siberian Mechanical Plant, Russia) made of nickel foil located in reactor 1. The temperature of the reactor was determined and controlled using systems 9 and 10 (ManoTom, Russia). The gas mixture leaving reactor 1 was purified from unreacted with rutile with a fluorine concentrate and separated in a condenser 16 (Siberian Mechanical Plant, Russia) and a sorption column 17 (Siberian Mechanical Plant, Russia). The concentration of fluorine and gaseous fluorides in the gas phase removed to the ventilation system was determined by infrared spectrometry.

For this, a continuous method based on the interaction of fluorine with sulfur dioxidewas used:

$$
\mathrm{SO}_{2}+\mathrm{F}_{2} \rightarrow \mathrm{SO}_{2} \mathrm{~F}_{2}
$$

Sulfuryl fluoride formed in this process was analyzed by infrared spectroscopy on a 4300 DTGS spectrometer (Agilent Technologies, Santa Clara, CA, USA) in a continuous mode.

\section{Results}

The thermodynamic study of the fluorination of rutile ore concentrates was conducted at temperatures from $300 \mathrm{~K}$ to $1800 \mathrm{~K}$. For the main reaction of interest is the reaction between $\mathrm{TiO}_{2}$ and $\mathrm{F}_{2}$, at different temperatures. The process that took place was described in detail in our previous Procedia Chemistry conference report [19] with the main reactions of the process including:

$$
\mathrm{TiO}_{2 \text { (solid) }}+2 \mathrm{~F}_{2 \text { (gas) }} \rightarrow \mathrm{TiF}_{4 \text { (gas) }}+\mathrm{O}_{2 \text { (gas) }}
$$


In addition, reactions occur between $\mathrm{F}_{2}$ and other components of the original rutile concentrate:

$$
\begin{gathered}
\mathrm{SiO}_{2}+2 \mathrm{~F}_{2} \rightarrow \mathrm{SiF}_{4} \uparrow+\mathrm{O}_{2} \\
\mathrm{MgO}+\mathrm{F}_{2} \rightarrow \mathrm{MgF}_{2} \downarrow+0.5 \mathrm{O}_{2} \\
\mathrm{MnO}+\mathrm{F}_{2} \rightarrow \mathrm{MnF}_{2} \downarrow+0.5 \mathrm{O}_{2} \\
\mathrm{Al}_{2} \mathrm{O}_{3}+3 \mathrm{~F}_{2} \rightarrow 2 \mathrm{AlF}_{3} \downarrow+1.5 \mathrm{O}_{2} \\
\mathrm{FeO}+1.5 \mathrm{~F}_{2} \rightarrow \mathrm{FeF}_{3} \downarrow+0.5 \mathrm{O}_{2} \\
\mathrm{Fe}_{2} \mathrm{O}_{3}+3 \mathrm{~F}_{2} \rightarrow 2 \mathrm{FeF}_{3} \downarrow+1.5 \mathrm{O}_{2} \\
\mathrm{CaO}+\mathrm{F}_{2} \rightarrow \mathrm{CaF}_{2} \downarrow+0.5 \mathrm{O}_{2} \\
\mathrm{~V}_{2} \mathrm{O}_{5}+5 \mathrm{~F}_{2}=2 \mathrm{VF}_{5} \uparrow+2.5 \mathrm{O}_{2}
\end{gathered}
$$

The dependence of the change of the logarithmic equilibrium constant of these reactions on the temperature $\lg k p=f(T)$ was presented in Figure 2. According to the obtained results of thermodynamic equilibrium calculations, the fluorination of components in the ilmenite concentrate took place easily, without limitation, and the reverse oxidation to produce oxides did not happen. The main products formed in this process are fluoride salts. The composition and structure of an isolated thermodynamic system at the equilibrium moment was determined and evaluated using the ASTRA software (version 1.06, Moscow State Technical University N.E. Bauman, Russia).

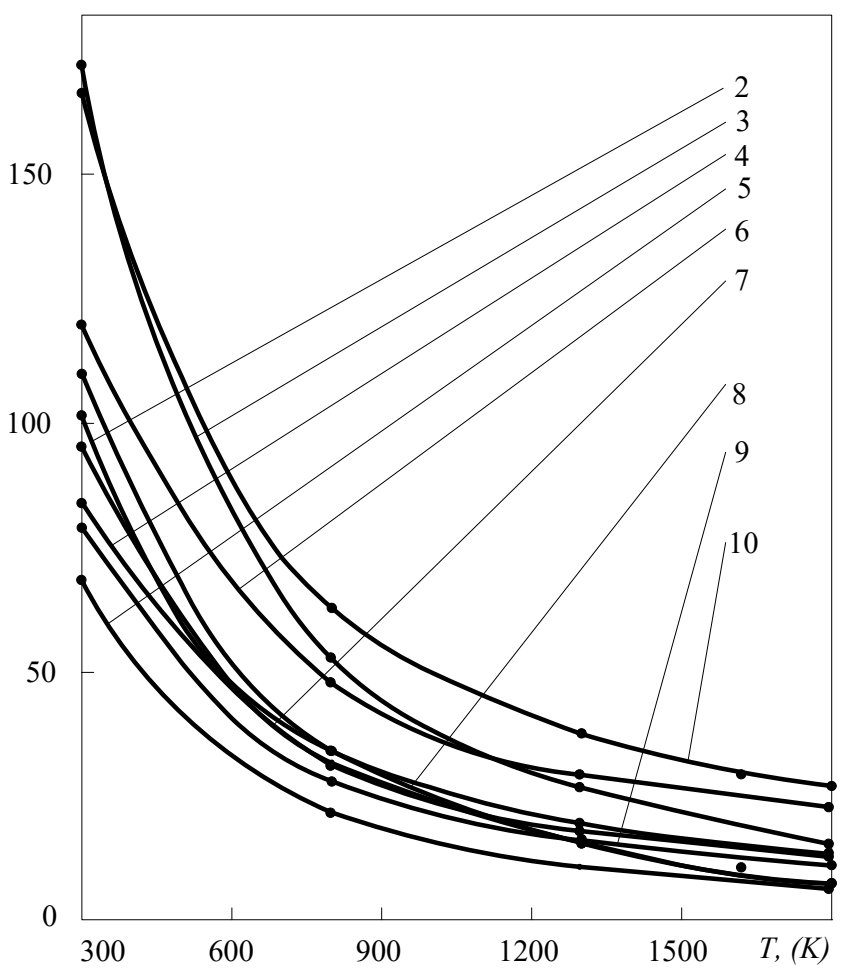

Figure 2. Effect of temperature reaction on $\lg k_{\mathrm{P}, \mathrm{T}}$ with different forming products. $2-\mathrm{TiF}_{4}, 3-\mathrm{SiF}_{4}$, $4-\mathrm{CaF}_{2}, 5-\mathrm{AlF}_{3}, 6-\mathrm{MnF}_{2}, 7-\mathrm{MgF}_{2}, 8-\mathrm{FeF}_{3}$ (from $\mathrm{FeO}$ ), 9- $\mathrm{FeF}_{3}$ (from $\mathrm{Fe}_{2} \mathrm{O}_{3}$ ), $10-\mathrm{VF}_{5}$.

To evaluate the dependence of the $\mathrm{TiF}_{4}$ content obtained in the product on the temperature, the experiments were carried out with the temperature from 300 to $1000 \mathrm{~K}$, under the condition of $10 \% \mathrm{~F}_{2}$ excess, $50 \% \mathrm{~F}_{2}$ excess, and $10 \% \mathrm{~F}_{2}$ deficiency and $50 \% \mathrm{~F}_{2}$ deficiency, corresponding to the ratio $\mathrm{TiO}_{2} / \mathrm{F}_{2}$ by mass: The results were shown in Figure 3. Here, the observed target products are $\mathrm{TiF}_{4}$ and $\mathrm{O}_{2}$, for other substances with high content. In total, $<0.0001 \mathrm{~mole} / \mathrm{kg}$ were removed from the calculation results. The need to conduct 
research under conditions of $10 \%$ and $50 \%$ fluorine deficiency is determined by the fact that in industrial conditions the fluorination process is carried out in two stages: the first-in an excess of fluorine, the second-in a fluorine deficiency. This is necessary to ensure the full use of fluorine in the process. In this case, at the first stage, the completeness of the fluorination of the rutile concentrate is ensured. For this, it is necessary to provide an excess of fluorine. In the second stage, it is necessary to capture the excess fluorine from the first stage. For this, the solid phase (rutile concentrate) is supplied in excess relative to the amount of fluorine leaving the first stage. As a result, titanium oxyfluorides and other components (solids) are formed, which are then returned to the first stage together with the rutile concentrate. From Figure 3, it could be seen that a higher $\mathrm{TiF}_{4}$ content was observed in the equilibrium ratio of the phases under ideal conditions, however, in the system, there was still unreacted $\mathrm{TiO}_{2}$ (analyzed by solid phase XRD). The concentration of $\mathrm{TiF}_{4}$ (gas) was determined in the temperature range from $400 \mathrm{~K}$ to $600 \mathrm{~K}$. Its value increased from $0.05 \%$ by mass to $\sim 75 \%$ by mass under ideal conditions (with a large excess of $\mathrm{F}_{2}$ ), and with a $10 \%$ excess of $F_{2}$ it was approximately $72 \%$. When the temperature reaction was higher $600 \mathrm{~K}$, the concentration of $\mathrm{TiF}_{4}$ was stable and not changing. In the products, at both $400 \mathrm{~K}$ and $600 \mathrm{~K}$, there is a small amount of $\mathrm{V}_{2} \mathrm{O}_{5}$ (solid) approximately $0.053 \%$ by mass and theunreacted gas $\mathrm{F}_{2} \sim 4.5 \%$. Therefore, the optimal condition for carrying out fluorination (at first stage) is $10 \%$ excess $\mathrm{F}_{2}$ of its amount required for carrying out reactions (2-10).Thus, the amount of $\mathrm{F}_{2}$ required for fluorination should not exceed $10 \%$. This corresponds to the initial content of substances used in the main process equal to $\%$ (mass): $\mathrm{TiO}_{2}-48.43$ and $\mathrm{F}_{2}-53.63$.

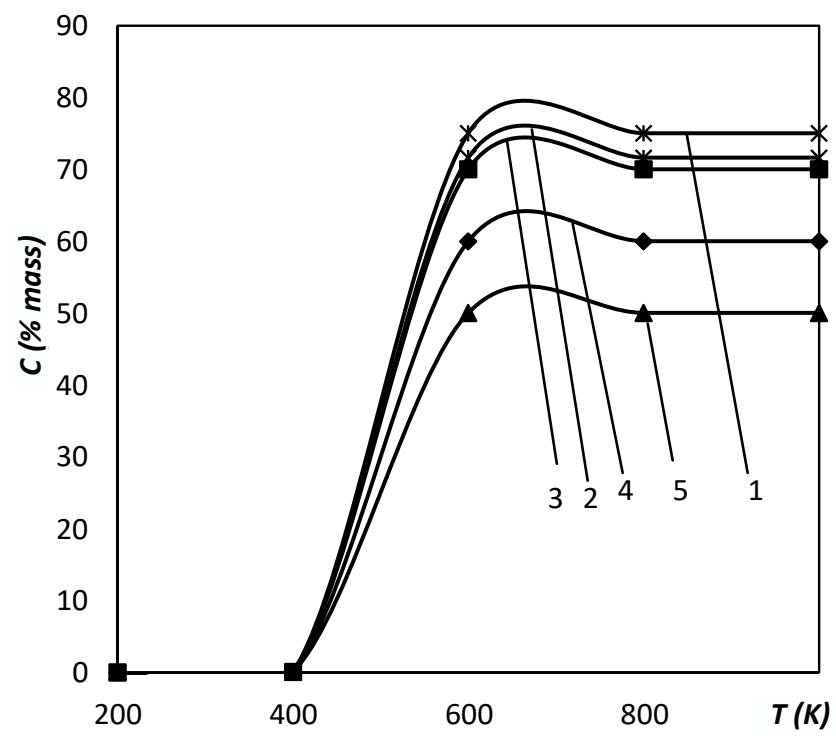

Figure 3. Effect of reaction temperature on concentration of $\mathrm{TiF}_{4}$ in the product mixture [19]. 1 -Ideal condition according to equilibrium measurement; 2 -excess of $10 \% \mathrm{~F}_{2} ; 3$-excess of $50 \% \mathrm{~F}_{2}$; 4 -deficiency of $10 \% \mathrm{~F}_{2}$ and 5-deficiency of $50 \% \mathrm{~F}_{2}$.

It can be seen from the results obtained that: $\mathrm{TiF}_{4}$ (gas) begins to form at a reaction temperature of $>400 \mathrm{~K}$, and a significant amount of gaseous $\mathrm{TiF}_{4}$ begins to form at $500 \mathrm{~K}$. Therefore, the study of the process of rutile fluorination should be carried out at temperatures $>500 \mathrm{~K}$.

The kinetics of the process of fluorination between $\mathrm{TiO}_{2}$ with fluoride was studied on samples with a particle size from $2 \times 10^{-6}$ to $2 \times 10^{-5} \mathrm{~m}$ with the specific surface area of $\sim 3.5 \mathrm{~m}^{2} / \mathrm{g}$.

These studies were performed on $\mathrm{TiO}_{2}$ samples; where the weight of each sample is $\sim 280 \mathrm{mg}$ and thickness of up to $4 \mathrm{~mm}$. The temperature of the study was conducted in range from 300 to $550{ }^{\circ} \mathrm{C}$. During the kinetics study, the temperature, partial pressure and 
sample mass parameters are recorded continuously over time. The fluorine gas used in this study is technical fluorine purified from HF by adsorption at $370-380{ }^{\circ} \mathrm{C}$ on NaF material.

The fluorination reaction occurs on the contact surface of the solid phase (sample) and gaseous phases. The process consists of several main stages: (1) supply of fluorinating gas $\left(\mathrm{F}_{2}\right)$ to the surface of the solid phase $\left(\mathrm{TiO}_{2}\right) ;(2)$ adsorption of gas on the surface of solid particles; (3) the chemical reaction of the sorbed gas with a solid; (4) desorption of gaseous $\mathrm{TiF}_{4}$ from the surface of the solid product; (5) diffusion of the desorbed $\mathrm{TiF}_{4}$ product into the gas phase.

When studying the kinetics of heterogeneous processes, the most difficult is to choose a mathematical model to describe the process under consideration. To describe reactions proceeding according to a mechanism that assumes that the rate of interaction of all particles located both on the surface of the powder layer and in its volume is the same and the process proceeds with a decrease in the particle size, the equations of "contracting sphere", "contracting cylinder" are usually used, Kazeev-Erofeev, Yander, Gistling, Prout-Tomkins. For processes in which reactions develop in a branched mechanism, Prout-Tompkins et al. used equations [20-26].

The reaction rate of $\mathrm{TiO}_{2}$ with fluorine will be a function of the temperature of the process, the partial pressure of the gas $\mathrm{F}_{2}$ and the physico-chemical properties of the original rutile- $\mathrm{TiO}_{2}$ material. Qualitative observations have been established temporarily at temperatures of $500 \mathrm{~K}$ and below, the process rate is very slow, as suggested by the authors, possibly related to the formation of the intermediate product-titanium oxyfluoride. The first compound forms a film on the surface of the titanium dioxide particles, in fact the process is stopped, the reaction taking place at a remarkable rate only at $630 \mathrm{~K}$ and above. At $830 \mathrm{~K}$ the reaction rate increases gradually and the vaporization of titanium in the gaseous form of titanium tetrafluoride ends within 12 to $14 \mathrm{~min}$.

The experimental dataof the kinetic study are shown in Figure 4, which was carried out in the temperature range of $580-830 \mathrm{~K}$.

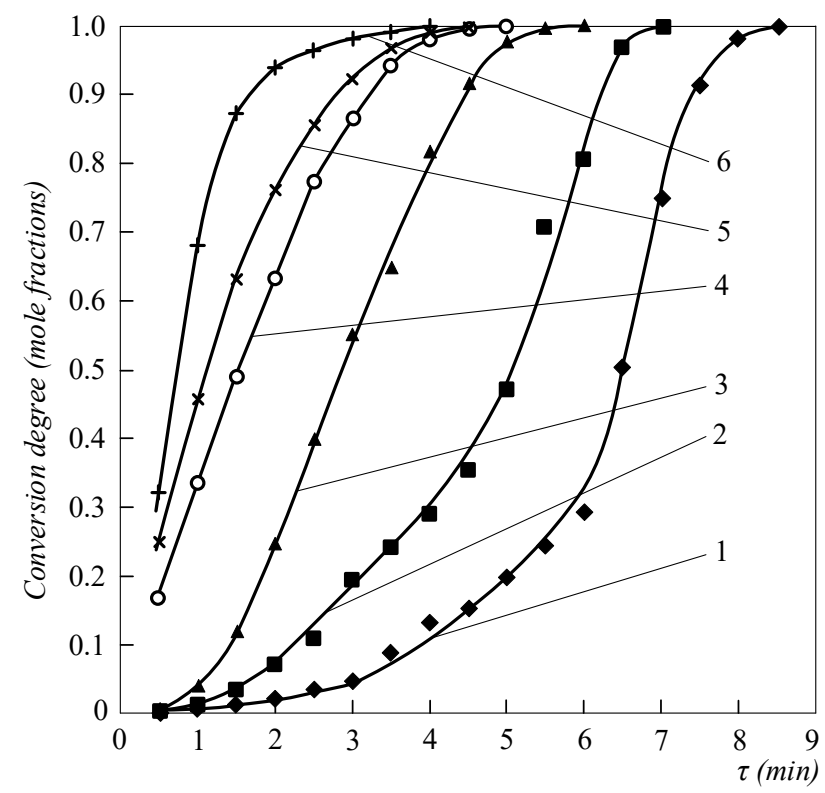

Figure 4. The effect of the time and temperature of fluorination on the degree of conversion in the titanium "dioxide-fluorine" system. 1-580; 2-630; 3-680; 4-730; 5-780; 6-830 K.

The obtained values of the accuracy of the approximation $R^{2}$, of the activation energy $E_{a}$, of the pre-exponential coefficient $k_{0}$ are presented in Table 1. 
Table 1. Values of activation energy and pre-exponential coefficient of the fluorination of titanium dioxide with elemental fluorine, calculated by various models.

\begin{tabular}{cccc}
\hline Models & $\boldsymbol{E}_{\boldsymbol{a}}(\mathrm{J})$ & $\boldsymbol{k}_{\mathbf{0}}\left(\mathbf{m i n}^{-1}\right)$ & $\begin{array}{c}\text { The Value of the Accuracy of } \\
\text { the Approximation } \mathbf{R}^{2}\end{array}$ \\
\hline $\begin{array}{c}\text { Gistling's equation } \\
1-2 \alpha / 3-(1-\alpha)^{2 / 3}=k \tau\end{array}$ & 4469.6 & 0.48 & 0.777 \\
\hline $\begin{array}{c}\text { Yander's equation } \\
\left(1-(1-\alpha)^{1 / 3}\right)^{2}=k \tau\end{array}$ & 3361.3 & 0.64 & 0.894 \\
\hline $\begin{array}{c}\text { (reduced sphere» equation } \\
1-(1-\alpha)^{1 / 3}=k \tau\end{array}$ & 4654.2 & 0.72 & 0.995 \\
\hline
\end{tabular}

The closest to unity value of the reliability of approximation $R^{2}$ was obtained using the equation of a "reduced sphere".

If the assumption about the fluorination of a spherical particle of the rutile concentrate is valid, then in the coordinates time $-1-(1-\alpha)^{1 / 3}$ the experimental points should lie on a straight line. The time dependences of $1-(1-\alpha)^{1 / 3}$ changes at different temperatures are shown in Figure 5.

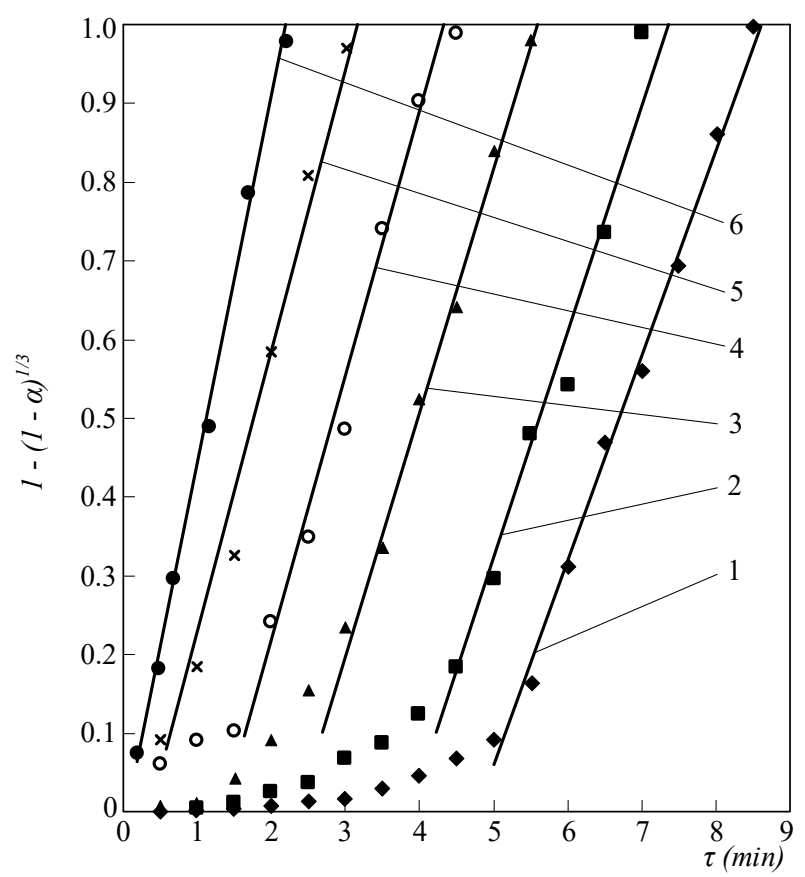

Figure 5. Dependence $1-(1-\alpha)^{1 / 3}$ on time at fluorination temperatures: $1-580 ; 2-630 ; 3-680$; 4-730; 5-780; 6-830 K.

From these data it can be seen that a directly proportional relationship is observed throughout the entire fluorination process, with the exception of the initial and final moments. The deviation from the straight-line relationship at the beginning of the process is due to the filling of the system with nitrogen, which is necessary to remove air from the system; in the last stage of the process, not the rutile concentrate is fluorinated, but the formed intermediate oxyfluorides of titanium and impurity elements of the rutile concentrate. Thus, the presented model of fluorination of a rutile concentrate particle describes well the process of its interaction with fluorine.

Using the data shown in Figure 5, the rate constants of the process of fluorination of rutile concentrate at various temperatures were determined and shown in Table 2. 
Table 2. Calculated kinetic values of the process of fluorination of rutile concentrate with elemental fluorine.

\begin{tabular}{cccc}
\hline $\boldsymbol{T}(\mathbf{K})$ & $\mathbf{1 0 0 0 / T}\left(\mathbf{K}^{-1}\right)$ & $k\left(\mathbf{m i n}^{-1}\right)$ & $\ln k$ \\
\hline 580 & 1.72 & 0.27 & -1.30 \\
\hline 630 & 1.39 & 0.29 & -1.22 \\
\hline 680 & 1.30 & 0.32 & -1.15 \\
\hline 730 & 1.22 & 0.34 & -1.09 \\
\hline 780 & 1.18 & 0.35 & -1.05 \\
\hline 830 & 1.14 & 0.36 & -1.02 \\
\hline
\end{tabular}

\section{Discussion}

The kinetic curves of the fluorination process of rutile were S-shaped (Figure 4), characteristic of heterogeneous gas-solid processes [23-25]. Three areas could be distinguished on the kinetic curves and include:

1. At stage 1, an induction stage characterized by a low velocity and associated with the diffusion of the initial titanium dioxide into intermediate product-titan oxyfluorides. In this stage, the formation of the phase interface, consisting of an intermediate compound (oxyfluoridation), took place. Fluorine atoms diffuse into the original solid reagent, becoming cores for intermediate compounds.

2. At stage 2, as new cores form, the rate of the process increases, reaching a maximum value, and in this part of the kinetic curves there is determination by the advancement of the interface.

3. At stage 3-A high degree of conversion is achieved, the solid particles are reduced, the specific surface is reduced, so the conversion rate increases slowly-a decrease in the rate of conversion [25-29].

The kinetic curve of fluorination process at $580 \mathrm{~K}$ was characterized by a large induction stage. A significant increase in the rate of fluorination at $780 \mathrm{~K}$ and above to $830 \mathrm{~K}$ due to the fact that the reaction switches to combustion mode. It should be noted that fluorination releases considerable heat, making it difficult to maintain isothermal conditions of the process.

The effect of reaction time on fluorination process according to the "reduced sphere" equation was showed in Figure 6 (temperature range from 580 to $830 \mathrm{~K}$ ).

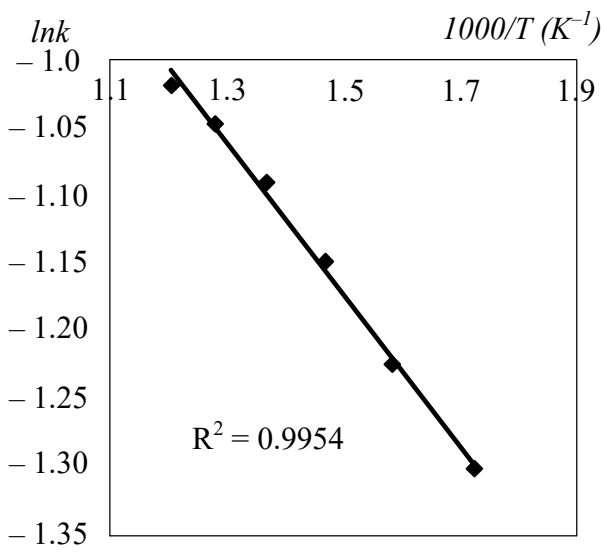

Figure 6. The effect of absolute temperature $(T, K)$ on $\ln k$ for the "reduced" sphere equation.

Temperature influence on the process rate of the fluorination of concentrate rutile, being calculated by the "reduced sphere" equation, is shown in Figure 6. For the definition 
of process activation energy $\left(E_{a}\right)$ performed linearization of experimental points in graph with coordinates $\ln k$ and $1 / T$.

If the beginning and the end of the process are considered separately, then a linear dependence is maintained throughout the entire process. In the first stage, there is a deviation due to inert gas-nitrogen in the system to remove air, so the partial pressure of fluorine gas is not stable. In the final stage, mainly titanium oxyfluoride intermediate product is formed.

Based on the data in Figure 6, the seeming activation energy of the process $E_{a}$ (4654.2 Joule/mole) and the pre-exponential coefficient $\left(k_{0}=0.72 \mathrm{~min}^{-1}\right)$ have been calculated. The kinetic equation will then be represented:

$$
1-(1-\alpha)^{\frac{1}{3}}=k \cdot \tau=k_{0} \cdot e^{\frac{-E_{a}}{R T}} \cdot \tau=0.72 \cdot e^{\frac{-4654.2}{R T}} \cdot \tau
$$

The obtained activation energy values show that the fluorination process takes place in the second control stage-chemical adsorption of $\mathrm{F}_{2}$ gas on the solid phase surface-rutile. If the process takes place in the kinetic field, then $E_{a}$ must be greater than $40,000 \mathrm{~J} / \mathrm{mol}$.

It should be noted that all the mechanisms described above relate to the case of using a large excess of fluorine, although in practice a minimal excess is usually used. When receiving intermediate fluorides from rutile, the processing rate is high enough, and the $\mathrm{F}_{2}$ can be used almost completely. When titanium tetrafluoride is obtained from intermediate fluorides, it is possible to achieve quantitative use of fluoride only with a very long contact of the rutile and gas $\mathrm{F}_{2}$; this is possible in a counter-current process where fresh fluoride is supplied to the partially perfluorinated product and the off-gases are treated with fresh rutile.

In static conditions (or at the insufficient intensity of stirring) and at a high concentration of fluoride we observed significant overheating of a solid material. Since intermediate titanium oxyfluoride and admixtures, contained in the rutile concentrate, can sinter, the bad stirring of solid and gaseous phases leads to the fusion of material and to even greater deterioration of conditions of phases contact. Therefore, it is more favorable to carry out the process of titanium tetrafluoride formation in gaseous suspension. Under these conditions, the heating up of a particle is insignificant, as its temperature is practically equal to the gas temperature.

A schematic diagram of a facility for fluorination of rutile concentrate in industrial conditions is shown in Figure 7. Fluorination of rutile must be carried out in two stages. At the first stage, according to reactions (2-10), in an excess of elemental fluorine at 1500-2000 K, the process of fluorination of rutile concentrates proceeds.

At this stage, both volatile fluorides $\mathrm{TiF}_{4}, \mathrm{SiF}_{4}$, and $\mathrm{VF}_{5}$ and nonvolatile fluorides $\mathrm{MgF}_{2}, \mathrm{MnF}_{2}, \mathrm{AlF}_{3}, \mathrm{FeF}_{3}$, and $\mathrm{CaF}_{2}$ are formed. Elemental fluorine is an expensive reagent; therefore, in the process of fluorination, it is necessary to ensure conditions under which its losses are minimal, and the utilization rate is maximum. To ensure these conditions, the second stage of rutile fluorination is carried out - capturing the excess of elemental fluorine from the first stage of fluorination, proceeding according to the reaction:

$$
\mathrm{TiO}_{2} \text { excess }+2 \mathrm{~F}_{2} \rightarrow \mathrm{TiOF}_{2 \text { solid }}+\mathrm{O}_{2}
$$

In this case, in the process of interaction of elemental fluorine with $\mathrm{TiO}_{2}$, a nonvolatile compound, $\mathrm{TiOF}_{2}$ is formed, which ensures the full use of fluorine.

A two-stage scheme of the fluorination of rutile concentrates is shown in Figure 7.

From the upper part of reactor 6 for collecting elemental fluorine from the first stage of fluorination, the dust-gas phase enters filter 7 , designed to separate particles of the solid phase (dust) from the gas stream. Titanium tetrafluoride is desublimated from the gas phase purified from solid particles at $278{ }^{\circ} \mathrm{C}$ in desublimator 10 to form a solid phase-titanium tetrafluoride powder. The resulting $\mathrm{TiF}_{4}$ powder is collected in tank 11 . From this tank, titanium tetrafluoride is sent for further processing, for example, in the process of titanium powder synthesis. 


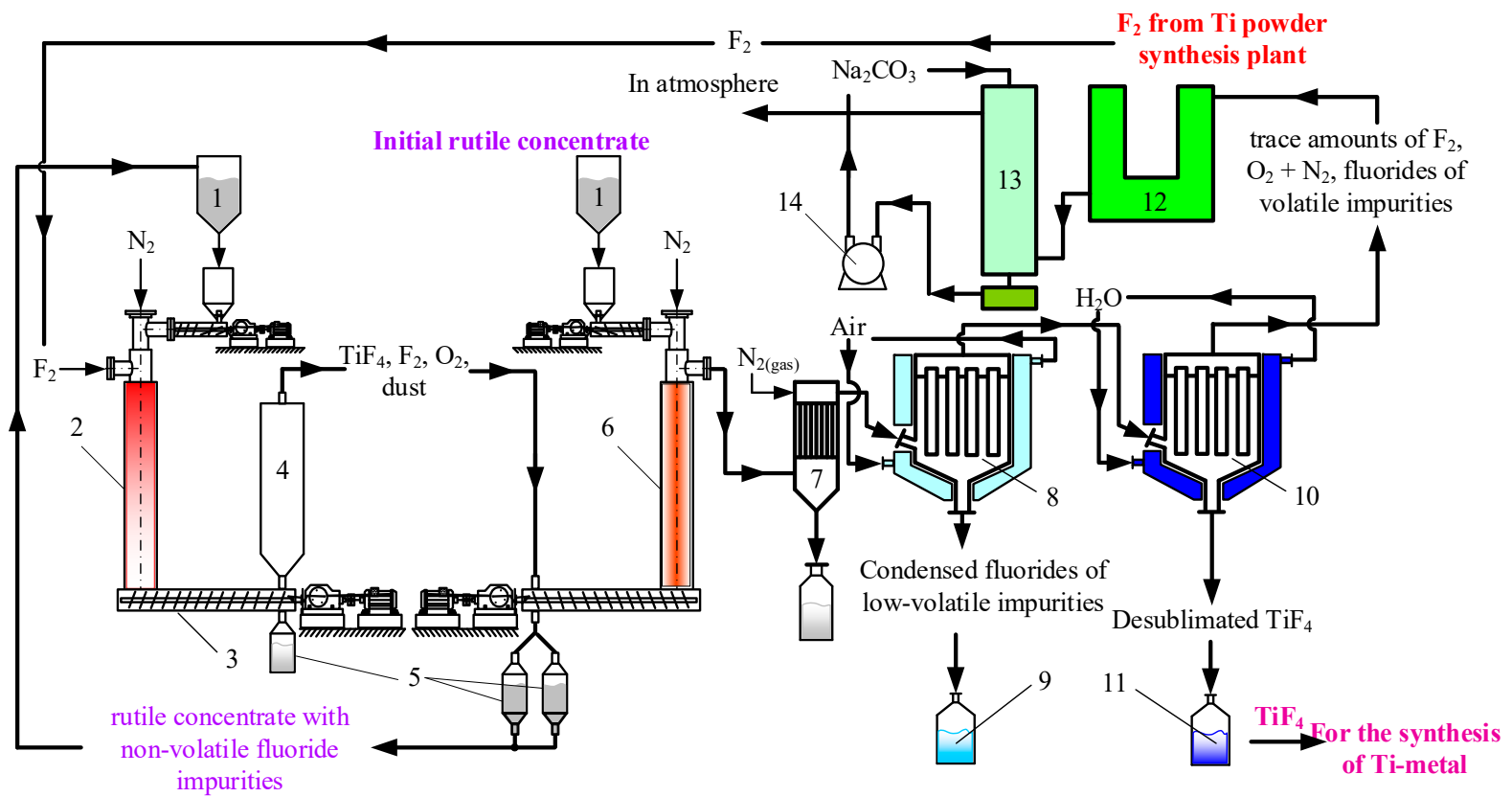

Figure 7. Apparatus and technological scheme for fluorination of rutile concentrate with elemental fluorine. 1-Bunker with the original rutile concentrate; 2-Flame reactor; 3-Reactor for unloading fluoride cinder; 4 - Container for the separation of non-volatile fluorides and non-fluorinated rutile concentrate; 5 -Bunkers for collecting solid phase; 6 - Reactor for capturing $\mathrm{F}_{2}$ from the first stage of fluorination; 7-Filter; 8-Filter-cooler of the gas phase; 9-Tank for condensed fluorides of lowvolatile impurities; $10-\mathrm{TiF}_{4}$ desublimator; 11 -Container for collecting $\mathrm{TiF}_{4}$ powder; $12-\mathrm{Scrubber}$ for cleaning fluorinated gases; 13-Column for post-treatment of waste gases from fluorine-containing components; 14-Pump.

The gas-phase leaving the desublimator 10 contains trace amounts of fluorine, oxygen and nitrogen. In order to ensure the possibility of removing this gas phase into the atmosphere, it is necessary to clean it from traces of fluorine. This process is carried out in two apparatuses-a scrubber for cleaning fluorinated gases 12 and a column 13 for additional purification of waste gases from fluorinated components with a 10\% sodium carbonate solution. As a result, a gas phase is obtained, in which a mixture of oxygen and nitrogen is obtained, in which the fluorine content does not exceed the limiting value, which allows this gas mixture to be discharged into the atmosphere.

\section{Conclusions}

Based on the studies performed on the substantiation of the process of fluorination of rutile concentrate with elemental fluorine, the following conclusions can be shown:

1. Methods of calculation using the isobar equation and high-temperature components of enthalpy $\left(\Delta \mathrm{H}^{\circ} \mathrm{T}^{\mathrm{T}}-\Delta \mathrm{H}^{\circ}{ }_{298}\right)$, and entropy $\left(\mathrm{S}^{\circ} \mathrm{T}^{-} \mathrm{S}^{\circ}{ }_{298}\right)$, as well as using the computer program "ASTRA". Thermodynamic calculations of the fluorination reactions of rutile concentrates by elemental fluorine, the dependences of the change in the equilibrium constant $(\ln K p)$ of the indicated reactions in the temperature range of $300-1800 \mathrm{~K}$ were determined.

2. As a result of thermodynamic analysis, it was found that when rutile concentrates interact with elemental fluorine, fluorination reactions in the temperature range under consideration proceed spontaneously with a large release of heat and do not have thermodynamic restrictions.

3. When rutile concentrates are processed with elemental fluorine, the system selfheating and the formation of volatile fluorides of titanium, silicon, vanadium and non-volatile fluorides of magnesium, manganese, aluminum, iron, calcium. 
4. Kinetic studies of the fluorination process of rutile concentrates with elemental fluorine, depending on temperature and pressure, have been carried out. Mathematical processing of quantitative data on their interaction of components according to the equations of Gistling, Yander and the "reduced" sphere was carried out.

It has been found that the process is most accurately described by the equation of a "reduced" sphere.

5. As a result, the value of the apparent activation energy $4654.2 \mathrm{~J} / \mathrm{mol}$ and the value of the pre-exponential coefficient $k_{0}=0.72 \mathrm{~min}^{-1}$ of the process of fluorination of titanium dioxide with elemental fluorine were determined. The kinetic equation for fluorination of concentrate rutile with elemental fluorine was found:

$$
1-(1-\alpha)^{\frac{1}{3}}=0.72 \cdot e^{\frac{-4654.2}{R T}} \cdot \tau
$$

This process takes place in the control-limited stage-chemical adsorption of $\mathrm{F}_{2}$ gas on the solid phase surface-rutilethermal equalization, and mass equalization fluorination reactions.

6. The obtained results allow to propose and consider the conditions of process execution on industrial equipment.

Author Contributions: Conceptualization. V.A.K.; methodology, H.M.T.L.; software, A.V.S.; validation, V.A.K.; investigation, A.N.S. and A.V.S.; resources, N.V.K. and A.N.S.; data curation, S.H.L.; writing-original draft preparation, N.V.K. and S.H.L.; writing-review and editing, H.M.T.L.; supervision, V.A.K.; project administration, V.A.K.; funding acquisition, H.M.T.L. All authors have read and agreed to the published version of the manuscript.

Funding: This research was funded and supported by the CE Program of Tomsk Polytechnic University, Vietnam Atomic Energy Institute and Vietnam Titanium Institute of Science and Technologyunder Hung Thinh Titanium Slag Factory Limited Company.

Institutional Review Board Statement: Not applicable.

Informed Consent Statement: Not applicable.

Data Availability Statement: Not applicable.

Acknowledgments: CE Program of Tomsk Polytechnic University and Vietnam Atomic Energy Institute supported this research.

Conflicts of Interest: The authors declare no conflict of interest. The funders had no role in the design of the study; in the collection, analyses, or interpretation of data; in the writing of the manuscript, or in the decision to publish the results.

\section{References}

1. Kraft, E. Summary of Emerging Titanium Cost Reduction Technologies; EHK Technologies for ORNL: Vancouver, BC, Canada, 2004; pp. 12-28.

2. Kroll, W.J. Ductile titanium. Trans. Electrochem. Soc. 1940, 112, 35-47. [CrossRef]

3. Fray, D.; Schwandt, C. Aspects of the Application of Electrochemistry to the Extraction of Titanium and Its Applications. Mater. Trans. 2017, 58, 306-312. [CrossRef]

4. Fray, D.J. Novel methods for the production of titanium. Int. Mater. Rev. 2008, 53, 317-325. [CrossRef]

5. Rodriguez, M.H.; Resales, G.D.; Pinna, E.G.; Tunez, F.M.; Toro, N. Extraction of Titanium from Low-Grade Ore Different Leaching Agents in Autoclave. Metals 2020, 10, 497. [CrossRef]

6. Dubenko, A.V.; Nikolenko, M.V.; Pasenko, O.O.; Kostyniuk, A.; Likozar, B. Intensification of Sulfuric Acid Leaching of Altered Ilmenite via Adding Fluoride Activator. Processes 2021, 9, 1922. [CrossRef]

7. Dubenko, A.V.; Nikolenko, M.V.; Kostyniuk, A.; Likozar, B. Sulfuric Acid Leaching of Altered Ilmenite Using Thermal, Mechanical and Chemical Activation. Minerals 2020, 20, 538. [CrossRef]

8. Dubenko, A.V.; Nikolenko, M.V.; Aksenenko, E.V.; Kostyniuk, A.; Likozar, B. Mechanism, Thermodynamics and Kinetics of Rutile Leaching Process by Sulfuric Acid Reactions. Processes 2020, 8, 640. [CrossRef]

9. Sachkov, V.I.; Nefedov, R.A.; Orlov, V.V.; Medvedev, R.O.; Sachkova, A.S. Hydrometallurgical Processing Technology of Titanomagnetite Ores. Minerals 2018, 8, 2. [CrossRef] 
10. Gordienko, P.S.; Dostovalov, V.A.; Pashnina, E.V. Hydrofluoride Method of Complex Processing of Titanium-Containing Raw Materials. Solid State Phenom. 2017, 265, 542-547. [CrossRef]

11. Gupta, A.K.; Aula, M.; Pihlasalo, J.; Makela, P.; Huttula, M.; Fabritius, T. Preparation of Synthetic Titania Slag Relevant to the Industrial Smelting Process Using an Induction Furnace. Appl. Sci. 2021, 11, 1153. [CrossRef]

12. Chen, G.; Ling, Y.; Li, Q.; Zheng, H.; Jiang, Q.; Li, K.; Gao, L.; Omran, M.; Chen, J. Highly efficient oxidation of Panzhihua titanium slag for manufacturing welding grade rutile titanium dioxide. J. Mater. Res. Technol. 2020, 9, 7079-7086. [CrossRef]

13. Chen, G.Z.; Fray, D.J.; Farthing, T. Direct electrochemical reduction of titanium dioxide to titanium in molten calcium chloride. Nature 2000, 407, 361-364. [CrossRef]

14. Jiao, S.; Zhu, H. Novel Metallurgical Process for Titanium Production. J. Mater. Res. 2006, 21, 2172-2175. [CrossRef]

15. Okabe, T.H.; Oda, T.; Mitsuda, Y. Titanium powder production by preform reduction process (PRP). J. Alloys Compd. 2004, 364, 156-163. [CrossRef]

16. Suzuki, R.O.; Teranuma, K.; Ono, K. Calciothermic reduction of titanium oxide and in-situ electrolysis in molten CaCl $\mathrm{Metall}$ Mater. Trans. 2003, 34, 287-295. [CrossRef]

17. Krishnan, A.; Lu, X.G.; Pal, U.B. Solid Oxide Membrane (SOM) technology for environmentally sound production of tantalum metal and alloys from their oxide sources Scand. J. Metall. 2005, 34, 293-301. [CrossRef]

18. Karelin, V.A.; Karelin, A.I. Fluoride Technology of Recycling the Concentrates of Rare Metals; Scientific and Technical Literature Publishing House: Tomsk, Russia, 2004.

19. Karelin, V.A.; Strashko, A.N.; Dubrovin, A.V.; Saronov, A.V. Research of fluorination process of rutile concentrate. Proc. Chem. 2014, 11, 56-62. [CrossRef]

20. Ono, K.; Okabe, T.H.; Suzuki, R.O. Design, Test and Theoretical Assessments for Reduction of Titanium Oxide to Produce Titanium in Molten Salt. Mater. Trans. 2017, 58, 313-318. [CrossRef]

21. Pal, U.B.; Woolley, D.E.; Kenney, G.B. Emerging SOM technology for the green synthesis of metals from oxides. Jom 2001, 53, 32-35. [CrossRef]

22. Scherbakov, V.I.; Zuev, V.A.; Parfyonov, A.V. Kinetics and Mechanism of Fluorination of Compounds of Uranium, Plutonium and Neptunium by Fluoride and Halogen Fluorides; Energoatomizdat: Moscow, Russia, 1985.

23. Levenshpil, O. Processes Design and Development Vol 7, № 4; ACS Publications: Washington, DC, USA, 1968 ; pp. 121-140.

24. Kopyrin, A.A.; Karelin, A.I.; Karelin, V.A. Technology of Manufacture and Radiochemical Recycling of Nuclear Fuel; Joint-Stock Co. Publishing House Atomenergoizdat: Moscow, Russia, 2006.

25. Krishnan, A.; Lu, X.G.; Pal, U.B. Solid oxide membrane process for magnesium production directly from magnesium oxide. Metall. Mater. Trans. 2005, 36, 463-473. [CrossRef]

26. Allanore, A.; Yin, L.; Sadoway, D.R. A new anode material for oxygen evolution in molten oxide electrolysis. Nature 2013, 497, 353-356. [CrossRef]

27. Chen, G.Z.; Fray, D.J.; Farthing, T.W. Cathodic deoxygenation of the alpha case on titanium and alloys in molten calcium chloride. Metall. Mater. Trans. 2001, 32, 1041-1052. [CrossRef]

28. Schwandt, C.; Fray, D.J. Determination of the kinetic pathway in the electrochemical reduction of titanium dioxide in molten calcium chloride. Electrochim. Acta 2005, 51, 66-76. [CrossRef]

29. Iwahara, H.; Esaka, T.; Mangahara, T. Mixed conduction and oxygen permeation in the substituted oxides for $\mathrm{CaTiO}_{3}$. J. Appl. Electrochem. 1988, 18, 173-177. [CrossRef] 